\title{
Searches for New Physics at HERA
}

\author{
Ioannis Gkialas \\ University of Aegean, Chios, Greece
}

\begin{abstract}
.
Recent results on physics beyond the standard model from ZEUS and H1 experiments are presented, in particlular results on single top and stop production, new currents modifying Standard Model (SM) Deep Inelastic Scattering (DIS) cross sections (eg. RH currents), low SM cross section processes and many possible final states, eg. multilepton and isolated lepton production, single top production, stop production, etc. The data samples were obtained during the older HERA runs as well as during the upgraded HERA-II phase started in 2003. In general the obtained results agree with standard model predictions. New particles have not been seen. H1 has seen an excess of diand tri-lepton events in the $e^{+} p$ channel.
\end{abstract}

Keywords: HERA ZEUS H1 BSM stop leptoquark

PACS: 12.38.-t, 12.20.Fv, 12.60.-i, 12.60.Jv, 14.80.Ly

\section{INTRODUCTION}

HERA is a $27.5 \mathrm{GeV}$ electron/positron on $820 \mathrm{GeV}$ proton collider. The main process involves the exchange of a photon between the incoming lepton and a quark in the proton. Typical final state involves the scattered final state electron, the current jet produced by the hit quark and the target jet in the proton beam direction originating from the diquark system resulting from the proton breakup. H1 and ZEUS are two general purpose detectors built around a liquid Ar and a compensating depleted Uranium - scintillator calorimeters respectively.

Following significant detector and luminosity upgrades put into effect in 2002 and 2003, large backgrounds were identified and overcome successfully. Efficient data taking started in October 2003 and a long run is scheduled until summer 2007. HERA-II can also provide polarized $e^{-} / e^{+}$beams using spin rotators. The transverse polarisation $P=\frac{N_{\uparrow}-N_{\downarrow}}{N_{\uparrow}-N_{\downarrow}}$ builds up naturally (Sokolov-Ternov effect). Spin rotators flip the polarisation by $90^{\circ}$ just before the lepton beam enters the interaction regions of experiments. Typical polarisation values reach values of $\approx 40 \%$. HERA-I has accumulated int $L \approx 130 \mathrm{pb}^{-1}$ while HERA-II has accummulated int $L \approx 230 \mathrm{pb}^{-1}$ until summer 2006.

\section{RESULTS}

\section{New currents}

In SM righthanded electrons do not exist $\sigma(R H)=0$. The same holds for lefthanded positrons, $\sigma(L H)=0$. The total cross-section changes linearly with polarisation. $\mathrm{H} 1$ and ZEUS results are consistent with SM predictions as it can be seen in figure 1 


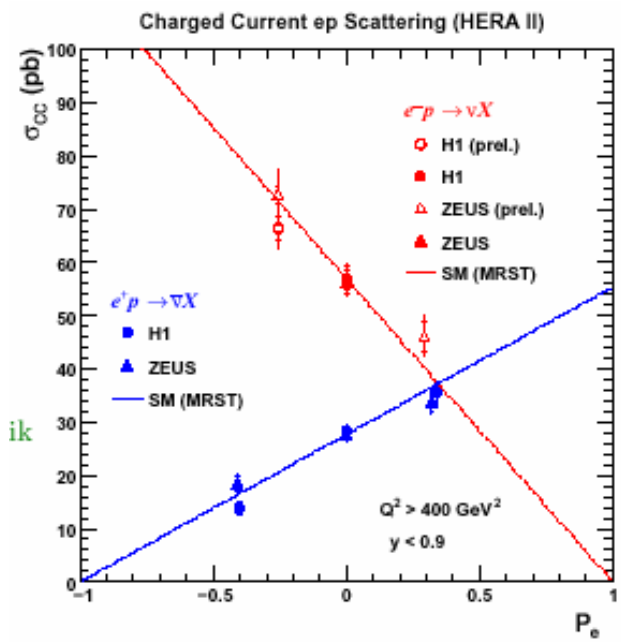

FIGURE 1. Total CC cross section $\left(Q^{2}>400 \mathrm{GeV}^{2}\right)$ vs. polarisation

\section{Leptoquarks}

Search for new particles in HERA can be done in single particle production mode. Interpretation though depends on couplings to SM particles. No absolute mass limits are produced. Leptoquarks are predicted by several beyond standard model (BSM) theories They connect lepton and quark sectors and carry both baryon and lepton quantum numbers. In HERA they can be produced in the s-channel as a resonance. They are scalar or vector color triplet bosons. The experimental signature is a lepton and a jet in the final state. No leptoquarks have been seen so far. Limits have been set in 14 LQ types. There is still good discovery potential for some types in HERA-II.

\section{Multilepton final state}

In the standard model multilepton final states can be rarely produced through $\gamma \gamma$ interactions. In several BSM production mechanisms multilepton events can easily occur (eg. $\mathrm{H}^{++}$production). $\mathrm{H} 1$ has seen a consistent excess of multilepton events over SM prediction in positron data only. They have identified 4 events in $e^{+} p$ when $0.6 \pm 0.1$ was expected. ZEUS has not seen any discrepancies. In tables 1 and 2 the $\mathrm{H} 1$ results are presented for dilepton and all multi lepton final states respectively.

\section{Single lepton, single top production}

Many processes indicating new physics would demonstrate themselves with an isolated lepton in the final state. Supersymmetric processes (eg. ep $\rightarrow \tilde{e} \tilde{q} X)$, R-parity violating supersymmetric processes (eg. Stop production), or top quark production at a rate higher than predicted by SM would indicate themselves with an isolated lepton in the 
TABLE 1. H1 dilepton production with high $P_{T}$ for positron-proton and electron-proton data. All data between 1994 and 2005 havew been included

H1 Preliminary $275 \mathrm{pb}^{-1}(1994-2005)$

\begin{tabular}{|c|c|c|c|c|}
\hline Selection & Data & SM & Pair Production & NC-DIS + Compton \\
\hline \multicolumn{5}{|c|}{$e^{+} p$ collisions $\left(156 \mathrm{pb}^{-1}\right)$} \\
\hline ee $M_{12}>100 \mathrm{GeV}$ & 3 & $0.44 \pm 0.10$ & $0.29 \pm 0.09$ & $0.15 \pm 0.04$ \\
\hline$\mu \mu M_{\mu \mu}>100 \mathrm{GeV}$ & 0 & $0.03 \pm 0.02$ & $0.03 \pm 0.02$ & - \\
\hline $\mathrm{e} \mu M_{e \mu}>100 \mathrm{GeV}$ & 0 & $0.29 \pm 0.03$ & $0.29 \pm 0.03$ & - \\
\hline eee $M_{12}>100 \mathrm{GeV}$ & 3 & $0.29 \pm 0.06$ & $0.29 \pm 0.06$ & - \\
\hline $\mathrm{e} \mu \mu M_{e \mu}>100 \mathrm{GeV}$ & 1 & $0.04 \pm 0.01$ & $0.04 \pm 0.01$ & - \\
\hline $\mathrm{e} \mu \mu M_{\mu \mu}>100 \mathrm{GeV}$ & 1 & $0.015 \pm 0.007$ & $0.015 \pm 0.007$ & - \\
\hline \multicolumn{5}{|c|}{$e^{-} p$ collisions $\left(119 \mathrm{pb}^{-1}\right)$} \\
\hline ee $M_{12}>100 \mathrm{GeV}$ & 0 & $0.42 \pm 0.11$ & $0.23 \pm 0.06$ & $0.19 \pm 0.06$ \\
\hline$\mu \mu M_{\mu \mu}>100 \mathrm{GeV}$ & 0 & $0.02 \pm 0.02$ & $0.02 \pm 0.02$ & - \\
\hline $\mathrm{e} \mu M_{e \mu}>100 \mathrm{GeV}$ & 0 & $0.24 \pm 0.04$ & $0.24 \pm 0.04$ & 一 \\
\hline eee $M_{12}>100 \mathrm{GeV}$ & 0 & $0.18 \pm 0.05$ & $0.18 \pm 0.05$ & - \\
\hline $\mathrm{e} \mu \mu M_{e \mu}>100 \mathrm{GeV}$ & 0 & $0.03 \pm 0.01$ & $0.03 \pm 0.01$ & - \\
\hline $\mathrm{e} \mu \mu M_{\mu \mu}>100 \mathrm{GeV}$ & 0 & $0.004 \pm 0.003$ & $0.004 \pm 0.003$ & 一 \\
\hline
\end{tabular}

TABLE 2. H1 combination of di- and trilepton production with high $P_{T}$ for positron-proton and electron-proton data. All data between 1994 and 2005 have been included

H1 Preliminary $275 \mathrm{pb}^{-1}(1994-2005)$

\begin{tabular}{|c|c|c|c|c|}
\hline Selection & Data & SM & Pair Production & NC-DIS + Compton \\
\hline $\mathrm{e}^{+} \mathrm{p} \sum P_{T}>100 \mathrm{GeV}$ & 4 & $0.6 \pm 0.1$ & $0.49 \pm 0.09$ & $0.11 \pm 0.04$ \\
$\mathrm{e}^{-} \mathrm{p} \sum P_{T}>100 \mathrm{GeV}$ & 0 & $0.5 \pm 0.1$ & $0.37 \pm 0.10$ & $0.13 \pm 0.04$ \\
$\mathrm{All} \sum P_{T}>100 \mathrm{GeV}$ & 4 & $1.1 \pm 0.2$ & $0.86 \pm 0.18$ & $0.24 \pm 0.06$ \\
\hline
\end{tabular}

final state. In the case of top quark an enhancement could be achieved through flavor changing neutral current process (FCNC) which could be attributed to supersymmetry, multi-Higgs-doublet models, or exotic quarks (GUT, string theories, etc).

Flavor Changing Neutral Current events has a small cross section $(<1 \mathrm{fb})$ in the SM. If events at HERA are attributed to single top production then the process would proceed through anomalous couplings. Final state electron disappears down the beampipe in $65 \%$ of the events.

The single top signature would involve an isolated high energy lepton, significant missing transverse momentum, and a jet from the b-quark decay in the leptonic decay channel of the W. In the hadronic channel it would involve 3 jets with $M_{\text {dijet }} \approx M_{W}$ and $M_{\text {total }} \approx M_{\text {top }}$. The background in the leptonic channels is due to $2 \gamma$ processes, NC DIS and single $\mathrm{W}$ production. The main background is due to single vector boson production (SM cross section $\approx 1.1 \mathrm{pb}$ at $318 \mathrm{GeV}$ cms energy) In the hadronic channel the background comes from QCD processes. Neither of the experiments has seen a significant excess over SM predictions. In table 3 the ZEUS results are presented. 
TABLE 3. ZEUS results on Single lepton production

\begin{tabular}{|c|c|c|}
\hline Isolated e candidates & $12<P_{T}^{X}<25 \mathrm{GeV}$ & $P_{T}^{X}>25 \mathrm{GeV}$ \\
\hline ZEUS (prel.) $98-99 \mathrm{e}^{-} p\left(17 \mathrm{pb}^{-1}\right)$ & $1 / 0.23 \pm 0.06(67 \%)$ & $0 / 0.32 \pm 0.09(65 \%)$ \\
ZEUS (prel.) 04-05 $e^{-} p\left(126 \mathrm{pb}^{-1}\right)$ & $3 / 1.75_{-0.32}^{+0.36}(57 \%)$ & $3 / 2.54_{-0.45}^{+0.46}(51 \%)$ \\
\hline ZEUS (prel.) $99-00 \mathrm{e}^{+} p\left(66 \mathrm{pb}^{-1}\right)$ & $1 / 1.04 \pm 0.11(57 \%)$ & $1 / 0.92 \pm 0.09(79 \%)$ \\
ZEUS (prel.) $03-04 \mathrm{e}^{+} p\left(40 \mathrm{pb}^{-1}\right)$ & $0 / 0.46 \pm 0.10(64 \%)$ & $0 / 0.58_{-0.09}^{+0.08}(76 \%)$ \\
\hline \hline ZEUS (prel.) $98-05 \mathrm{e}^{-} p\left(143 \mathrm{pb}^{-1}\right)$ & $4 / 1.98_{-0.32}^{+0.36}(58 \%)$ & $3 / 2.86 \pm 0.46(53 \%)$ \\
ZEUS (prel.) $99-04 \mathrm{e}^{+} p\left(106 \mathrm{pb}^{-1}\right)$ & $1 / 1.50 \pm 0.15(59 \%)$ & $1 / 1.50_{-0.13}^{+0.12}(78 \%)$ \\
\hline \hline ZEUS (prel.) $98-05 \mathrm{e}^{ \pm} p\left(249 \mathrm{pb}^{-1}\right)$ & $5 / 3.48_{-0.36}^{+0.39}(58 \%)$ & $4 / 4.36 \pm 0.47(61 \%)$ \\
\hline \hline H1 (prel.) 1994-2005 $\mathrm{e}^{ \pm} p\left(279 \mathrm{pb}^{-1}\right)$ & - & $11 / 4.7 \pm 0.9(69 \%)$ \\
\hline
\end{tabular}

\section{Supersymmetric searches}

HERA is limited in the search for supersymmetric particles due to the restriction of pair-production of SUSY particles. However, for mechanisms allowing single supersymmetric particle production it could still further the experimental limits. R-parity is defined as $R_{P}=(-1)^{3 B+L+2 S}$ and it takes the value +1 for particles and -1 for sparticles.

$\mathrm{R}$-parity conservation implies pair production of supersymmetric particles and a stable LSP (cold dark matter candidate). R-parity violation leads to resonant supersymmetric particle production while supersymmetric particles can decay back to SM particles. Rparity violating terms are $W_{R_{P}}=\lambda_{i j k} L_{i} L_{j} \bar{E}_{k}+\lambda_{i j k}^{\prime} L_{i} Q_{j} \bar{D}_{k}+\lambda_{i j k}^{\prime \prime} U_{i} \bar{D}_{j} \bar{D}_{k}$ where the $\lambda^{\prime} s$ are dimensionless free parameters. For stop production in ep colliders (lightest Sparticle) $\lambda_{131}^{\prime} \neq 0$

Stop Branching ratios and neutralino, chargino and gluino masses are controlled by $\mu$ which mixes Higgs superfields, $M_{1}, M_{2}, M_{3}$, SUSY breaking parameters related to $\mathrm{U}(1), \mathrm{SU}(2), \mathrm{SU}(3)$ gauginos, the $\tan \beta$ and the $\lambda_{i j k}, \lambda_{i j k}^{\prime}, \lambda_{i j k}^{\prime \prime}$ There is good agreement between data and MC. No evidence of resonance has been seen with $M_{L X}>100 \mathrm{GeV}$ $\left(M_{L X}^{2}=2 E_{e}^{\text {beam }} \sum_{i} E+P_{z i}\right)$.

Model dependent limits on stop production were calculated for different values of SUSY parameters. The ZEUS limits were set for $100 \mathrm{GeV}<M_{2}<$ $300 \mathrm{GeV}($ step $30 \mathrm{GeV})$, and $-300 \mathrm{GeV}<\mu<300 \mathrm{GeV}($ step $20 \mathrm{GeV})$ The scenario where neutralino is not LSP and $M_{\chi^{0}}<30 \mathrm{GeV}$ (excl. by LEP) was not considered.

In figure 2.a the yellow region is excluded at 95\% C.L., and the red region is excluded in part of the SUSY parameters. More restrictive limit up to masses $\approx 240 \mathrm{GeV}$ could be set compared to low energy experiments (APV).

Limits were also set by ZEUS for the mSUGRA scenario. Stop, chargino and neutralino masses and BR are determined by the values of $m_{0}, m_{1 / 2}, \tan b, \operatorname{sign}(\mu)$. The limit calculation was based on a Bayesian approach. The red region in figure 2.b is excluded at $95 \%$ C.L. The exclusion limit in the plane $\left(m_{0}, m_{1 / 2}\right)$ was defined as the region of the plane for which $\mathrm{R}<0.05$, where $\mathrm{R}$ is the ratio between the signal and the SM likelihoods. In the blue region there are no solutions. The yellow region, $M_{\chi^{0}}<30 \mathrm{GeV}$ is already excluded by LEP. Limits can rule out a stop with mass up to $270 \mathrm{GeV}$ 

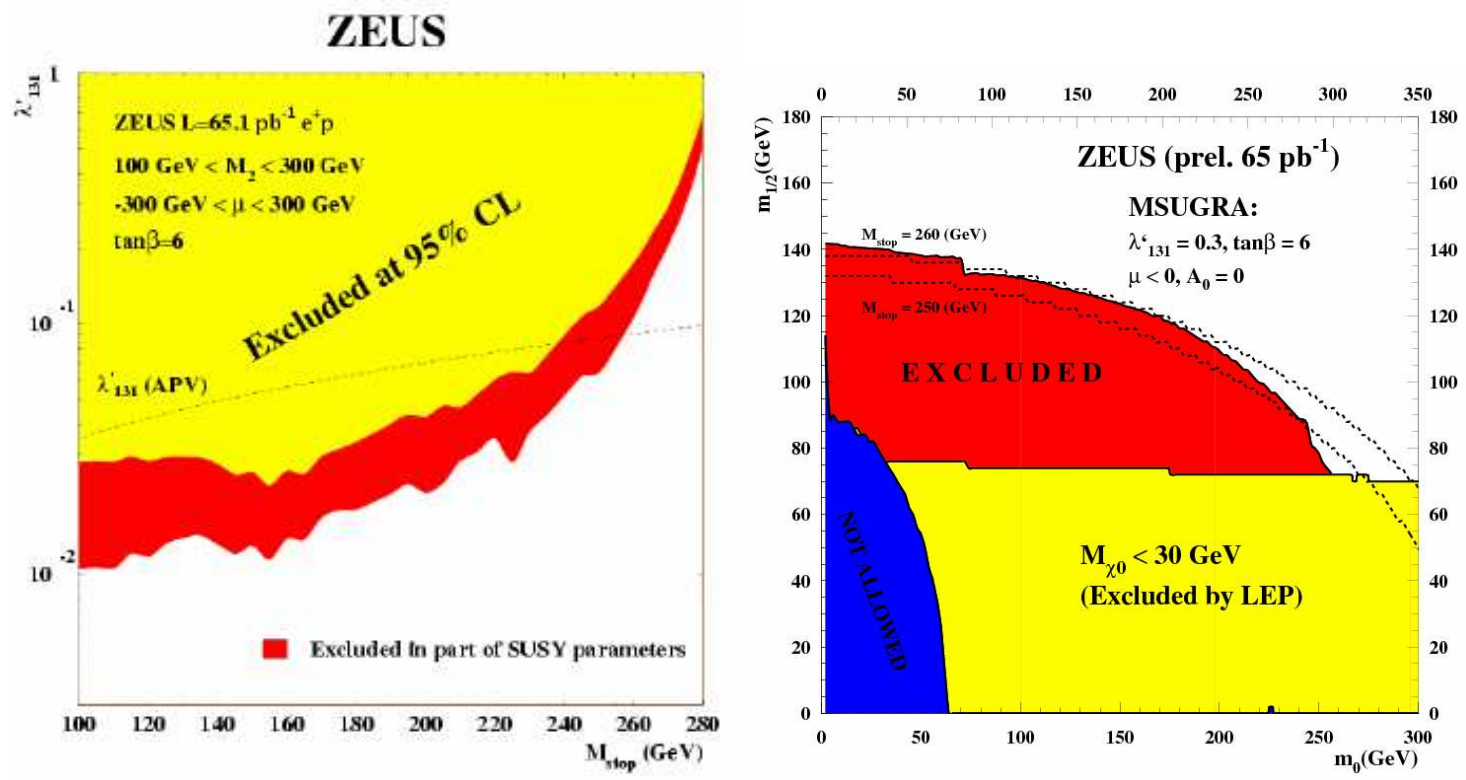

FIGURE 2. ZEUS results on stop production.(a) Minimal supersymmetric model (b) mSuGra

\section{CONCLUSIONS}

HERA II performs very well and the experiments $\mathrm{H} 1$ and ZEUS have been successfully upgraded. New limits on the parameter space of many BSM processes have been established by HERA. Results from HERA experiments are consistent with each other and with SM. There is however a persistent excess of events over the SM in high $P_{T}$ lepton searches observed by $\mathrm{H} 1$ in $e^{+} p$ collisions.

No evidence for resonances in the decay channels with jets and one high- $P_{T}$ lepton was found. Results were interpreted in terms of R-parity violating MSSM setting constraints on the Yukawa coupling $\lambda_{131}$ as a function of stop mass. Direct limits on the stop mass were derived within the mSUGRA model. For $\lambda_{131}=0.3, M_{\text {stop }}<270 \mathrm{GeV}$ are excluded for most of the parameter space. The set limit is more restrictive up to masses $\approx 240 \mathrm{GeV}$ compared to low energy experiments (APV). New data is expected at HERA II giving the possibility of pushing the limits of many BSM processes further

\section{ACKNOWLEDGMENTS}

This presentation has been possible through the financial endorsement of the Greek Ministry of Education, project EPEAEK. 